\title{
A Comparative Study of VIM and ADM for Solving Volterra-Fredholm Integro-Differential Equations
}

\author{
Ahmed A. Hamoud ${ }^{1 *}$, Nedal M. Mohammed ${ }^{2}$ and Kirtiwant P. Ghadle ${ }^{3}$ \\ ${ }^{1}$ Department of Mathematics, Taiz University, Taiz, Yemen \\ ${ }^{2}$ Department of Computer Science, Taiz University, Taiz, Yemen \\ ${ }^{3}$ Department of Mathematics, Dr. BAM University, Aurangabad-431004, India \\ *Corresponding author: drahmed985@yahoo.com
}

\begin{abstract}
In this article, we present a comparative study between the Adomian Decomposition Method (ADM) and Variational Iteration Method (VIM). The study outlines the significant features of the two methods, for solving nonlinear VolterraFredholm integro-differential equations. From the computational viewpoint, the VIM is more efficient, convenient and easy to use. Moreover, we proved the existence and uniqueness results and convergence of the solution. Finally, an example is included to demonstrate the validity and applicability of the proposed techniques.
\end{abstract}

Keywords: Adomian decomposition method; variational iteration method; VolterraFredholm integro-differential equation; approximate solution.

Mathematics Subject Classification (2010): 45J05, 49M27, 65K10.

\section{Introduction}

This paper outlines a reliable comparison between two powerful methods that were recently developed which are VIM and ADM. In this paper, we consider nonlinear VolterraFredholm integro-differential equation of the type

$$
\sum_{j=0}^{k} \xi_{j}(x) Z^{(j)}(x)=f(x)+\lambda_{1} \int_{a}^{x} K_{1}(x, t) G_{1}(Z(t)) d t+\lambda_{2} \int_{a}^{b} K_{2}(x, t) G_{2}(Z(t)) d t,
$$

with the initial conditions

$$
Z^{(r)}(a)=b_{r}, \quad r=0,1,2, \cdots,(k-1),
$$

where $Z^{(j)}(x)$ is the $j^{\text {th }}$ derivative of the unknown function $Z(x)$ that will be determined, $K_{i}(x, t), i=1,2$ are the kernels of the equation, $f(x)$ and $\xi_{j}(x)$ are analytic functions, $G_{1}$ and $G_{2}$ are nonlinear functions of $Z$ and $a, b, \lambda_{1}, \lambda_{2}$, and $b_{r}$ are real finite constants. In recent years, many authors focus on the development of numerical and analytical techniques for integro-differential equations. For instance, we can remember the following works. Abbasbandy and Elyas 2 studied some applications on variational iteration method for solving system of nonlinear Volterra integro-differential equations, Dawood et al. [8] applied the hybrid method for solving nonlinear Volterra-Fredholm integro-differential equations, Alao et al. [4] used ADM and VIM for solving integro-differential equations, Yang and Hou [17] applied the Laplace decomposition method to solve the fractional 
integro-differential equations, Mittal and Nigam [15] applied the ADM to approximate solutions for fractional integro-differential equations, and Behzadi et al. [6] solved some class of nonlinear Volterra-Fredholm integro-differential equations by homotopy analysis method. Moreover, several authors have applied the ADM and VIM to find the approx-

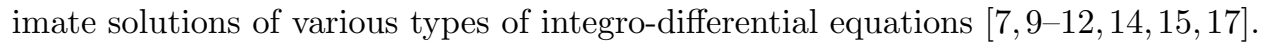

The main objective of the present paper is to study the behavior of the solution that can be formally determined by semi-analytical approximated methods as the ADM and VIM. Moreover, we proved the existence, uniqueness results and convergence of the solutions of the IVP (1)-(2).

\section{Description of the Methods}

Some powerful methods have been focusing on the development of more advanced and efficient methods for integro-differential equations such as the ADM [1, $3,5,12]$ and VIM $[2,4,13]$. We will describe these methods in this section:

\subsection{Description of the ADM}

Now, we can rewrite Eq.(1) in the form

$\xi_{k}(x) Z^{k}(x)+\sum_{j=0}^{k-1} \xi_{j}(x) Z^{j}(x)=f(x)+\lambda_{1} \int_{a}^{x} K_{1}(x, t) G_{1}(Z(t)) d t+\lambda_{2} \int_{a}^{b} K_{2}(x, t) G_{2}(Z(t)) d t$.

Then

$Z^{k}(x)=\frac{f(x)}{\xi_{k}(x)}+\lambda_{1} \int_{a}^{x} \frac{K_{1}(x, t)}{\xi_{k}(x)} G_{1}(Z(t)) d t+\lambda_{2} \int_{a}^{b} \frac{K_{1}(x, t)}{\xi_{k}(x)} G_{2}(Z(t)) d t-\sum_{j=0}^{k-1} \frac{\xi_{j}(x)}{\xi_{k}(x)} Z^{j}(x)$.

To obtain the approximate solution, we integrating $(k)$-times in the interval $[a, x]$ with respect to $x$ we obtain,

$$
\begin{aligned}
Z(x)= & L^{-1}\left(\frac{f(x)}{\xi_{k}(x)}\right)+\sum_{r=0}^{k-1} \frac{1}{r !}(x-a)^{r} b_{r}+\lambda_{1} L^{-1}\left(\int_{a}^{x} \frac{K_{1}(x, t)}{\xi_{k}(x)} G_{1}(Z(t)) d t\right) \\
& +\lambda_{2} L^{-1}\left(\int_{a}^{b} \frac{K_{2}(x, t)}{\xi_{k}(x)} G_{1}(Z(t)) d t\right)-\sum_{j=0}^{k-1} L^{-1}\left(\frac{\xi_{j}(x)}{\xi_{k}(x)} Z_{n}^{(j)}(x)\right)
\end{aligned}
$$

where $L^{-1}$ is the multiple integration operator given as follows:

$$
L^{-1}(\cdot)=\int_{a}^{x} \int_{a}^{x} \cdots \int_{a}^{x}(\cdot) d t d t \cdots d t \quad(k-\text { times }) .
$$

Now we apply ADM

$$
G_{1}(Z(x))=\sum_{n=0}^{\infty} A_{n}, G_{2}(Z(x))=\sum_{n=0}^{\infty} B_{n}
$$

where $A_{n}, B_{n} ; n \geq 0$ are the Adomian polynomials determined formally as follows:

$$
A_{n}=\left.\frac{1}{n !}\left[\frac{d^{n}}{d \mu^{n}} G_{1}\left(\sum_{i=0}^{\infty} \mu^{i} Z_{i}\right)\right]\right|_{\mu=0}, B_{n}=\left.\frac{1}{n !}\left[\frac{d^{n}}{d \mu^{n}} G_{2}\left(\sum_{i=0}^{\infty} \mu^{i} Z_{i}\right)\right]\right|_{\mu=0}
$$


The Adomian polynomials were introduced in $[16,17]$ as:

$$
\begin{array}{lll}
A_{0}=G_{1}\left(Z_{0}\right) ; & B_{0}=G_{2}\left(Z_{0}\right) \\
A_{1}=Z_{1} G_{1}^{\prime}\left(Z_{0}\right) ; & B_{1}=Z_{1} G_{2}^{\prime}\left(Z_{0}\right) ; \\
A_{2}=Z_{2} G_{1}^{\prime}\left(Z_{0}\right)+\frac{1}{2 !} Z_{1}^{2} G_{1}^{\prime \prime}\left(Z_{0}\right) ; & B_{2}=Z_{2} G_{2}^{\prime}\left(Z_{0}\right)+\frac{1}{2 !} Z_{1}^{2} G_{2}^{\prime \prime}\left(Z_{0}\right) \\
A_{3}=Z_{3} G_{1}^{\prime}\left(Z_{0}\right)+Z_{1} Z_{2} G_{1}^{\prime \prime}\left(Z_{0}\right)+\frac{1}{3 !} Z_{1}^{3} G_{1}^{\prime \prime \prime}\left(Z_{0}\right), & B_{3}=Z_{3} G_{2}^{\prime}\left(Z_{0}\right)+Z_{1} Z_{2} G_{2}^{\prime \prime}\left(Z_{0}\right)+\frac{1}{3 !} Z_{1}^{3} G_{2}^{\prime \prime \prime}\left(Z_{0}\right)
\end{array}
$$

The standard decomposition technique represents the solution of $Z$ as the following series:

$$
Z=\sum_{i=0}^{\infty} Z_{i} .
$$

By substituting (5) and (7) in Eq. (4) we have

$$
\begin{aligned}
\sum_{i=0}^{\infty} Z_{i}(x)= & L^{-1}\left(\frac{f(x)}{\xi_{k}(x)}\right)+\sum_{r=0}^{k-1} \frac{1}{r !}(x-a)^{r} b_{r}+\lambda_{1} \sum_{i=0}^{\infty} L^{-1}\left(\int_{a}^{x} \frac{K_{1}(x, t)}{\xi_{k}(x)} A_{i}(t) d t\right) \\
& +\lambda_{2} \sum_{i=0}^{\infty} L^{-1}\left(\int_{a}^{b} \frac{K_{2}(x, t)}{\xi_{k}(x)} B_{i}(t) d t\right)-\sum_{i=0}^{\infty} \sum_{j=0}^{k-1} L^{-1}\left(\frac{\xi_{j}(x)}{\xi_{k}(x)} Z_{i}^{(j)}(x)\right)
\end{aligned}
$$

The components $Z_{0}, Z_{1}, Z_{2}, \cdots$ are usually determined recursively by

$$
\begin{aligned}
Z_{0}= & L^{-1}\left(\frac{f(x)}{\xi_{k}(x)}\right)+\sum_{r=0}^{k-1} \frac{1}{r !}(x-a)^{r} b_{r} \\
Z_{1}= & \lambda_{1} L^{-1}\left(\int_{a}^{x} \frac{K_{1}(x, t)}{\xi_{k}(x)} A_{0}(t) d t\right)+\lambda_{2} L^{-1}\left(\int_{a}^{b} \frac{K_{2}(x, t)}{\xi_{k}(x)} B_{0}(t) d t\right) \\
& -\sum_{j=0}^{k-1} L^{-1}\left(\frac{\xi_{j}(x)}{\xi_{k}(x)} Z_{0}^{(j)}(x)\right), \\
Z_{n}= & \lambda_{1} L^{-1}\left(\int_{a}^{x} \frac{K_{1}(x, t)}{\xi_{k}(x)} A_{n-1}(t) d t\right)+\lambda_{2} L^{-1}\left(\int_{a}^{b} \frac{K_{2}(x, t)}{\xi_{k}(x)} B_{n-1}(t) d t\right) \\
& -\sum_{j=0}^{k-1} L^{-1}\left(\frac{\xi_{j}(x)}{\xi_{k}(x)} Z_{n-1}^{(j)}(x)\right), n \geq 1 .
\end{aligned}
$$

Then, $Z(x)=\sum_{i=0}^{n} Z_{i}$ as the approximate solution.

\subsection{Description of the VIM}

This method is applied to solve a large class of linear and nonlinear problems with approximations converging rapidly to exact solutions $[13,16]$. To illustrate, we consider the following general differential equation:

$$
L Z(t)+N Z(t)=f(t)
$$


where $L$ is a linear operator, $N$ is a nonlinear operator and $f(t)$ is inhomogeneous term. According to VIM [4], the terms of a sequence $Z_{n}$ are constructed such that this sequence converges to the exact solution. The terms $Z_{n}$ are calculated by a correction functional as follows:

$$
Z_{n+1}(t)=Z_{n}(t)+\int_{0}^{t} \lambda(\tau)\left(L Z_{n}(\tau)+N \tilde{y}(\tau)-f(\tau)\right) d \tau
$$

The successive approximation $Z_{n}(t), n \geq 0$ of the solution $Z(t)$ will be readily obtained upon using the obtained Lagrange multiplier and by using any selective function $Z_{0}$. The zeroth approximation $Z_{0}$ may be selected using any function that just satisfies at least the initial and boundary conditions. With $\lambda$ determined, several approximations $Z_{n}(t), n \geq 0$ follow immediately.

To obtain the approximation solution of IVP $(1)-(2)$, according to the VIM, the iteration formula 10 can be written as follows:

$$
\begin{aligned}
Z_{n+1}(x)= & Z_{n}(x)+L^{-1}\left[\lambda ( x ) \left[\sum_{j=0}^{k} \xi_{j}(x) Z_{n}^{(j)}(x)-f(x)-\lambda_{1} \int_{a}^{x} K_{1}(x, t) G_{1}\left(Z_{n}(t)\right) d t\right.\right. \\
& \left.\left.-\lambda_{2} \int_{a}^{b} K_{2}(x, t) G_{2}\left(Z_{n}(t)\right) d t\right]\right]
\end{aligned}
$$

where $L^{-1}$ is the multiple integration operator given as follows:

$$
L^{-1}(\cdot)=\int_{a}^{x} \int_{a}^{x} \cdots \int_{a}^{x}(\cdot) d x d x \cdots d x \quad(k-\text { times }) .
$$

To find the optimal $\lambda(x)$, we proceed as follows:

$$
\begin{aligned}
\delta Z_{n+1}(x)= & \delta Z_{n}(x)+\delta L^{-1}\left[\lambda ( x ) \left[\sum_{j=0}^{k} \xi_{j}(x) Z_{n}^{(j)}(x)-f(x)-\lambda_{1} \int_{a}^{x} K_{1}(x, t) G_{1}\left(Z_{n}(t)\right) d t\right.\right. \\
& \left.\left.-\lambda_{2} \int_{a}^{b} K_{2}(x, t) G_{2}\left(Z_{n}(t)\right) d t\right]\right] \\
= & \delta Z_{n}(x)+\lambda(x) \delta Z_{n}(x)-L^{-1}\left[\delta Z_{n}(x) \lambda^{\prime}(x)\right]
\end{aligned}
$$

From Eq. 12, the stationary conditions can be obtained as follows:

$$
\lambda^{\prime}(x)=0, \text { and } 1+\left.\lambda(x)\right|_{x=t}=0 .
$$

As a result, the Lagrange multipliers can be identified as $\lambda(x)=-1$ and by substituting in Eq. (11), the following iteration formula is obtained:

$$
\begin{aligned}
Z_{0}(x)= & L^{-1}\left[\frac{f(x)}{\xi_{k}(x)}\right]+\sum_{r=0}^{k-1} \frac{(x-a)^{r}}{r !} b_{r} \\
Z_{n+1}(x)= & Z_{n}(x)-L^{-1}\left[\sum_{j=0}^{k} \xi_{j}(x) Z_{n}^{(j)}(x)-f(x)-\lambda_{1} \int_{a}^{x} K_{1}(x, t) G_{1}\left(Z_{n}(t)\right) d t\right. \\
& \left.-\lambda_{2} \int_{a}^{b} K_{2}(x, t) G_{2}\left(Z_{n}(t)\right) d t\right], n \geq 0
\end{aligned}
$$


The term $\sum_{r=0}^{k-1} \frac{(x-a)^{r}}{r !} b_{r}$ is obtained from the initial conditions, $\xi_{k}(x) \neq 0$. Relation (13) will enable us to determine the components $Z_{n}(x)$ recursively for $n \geq 0$. Consequently, the approximation solution may be obtained by using

$$
Z(t)=\lim _{n \rightarrow \infty} Z_{n}(t)
$$

\section{Main Results}

In this section, we shall give an existence and uniqueness results of Eq. (1), with the initial condition (2) and prove it. We can be written Eq. (1) in the form of:

$$
\begin{aligned}
Z(x)= & L^{-1}\left[\frac{f(x)}{\xi_{k}(x)}\right]+\sum_{r=0}^{k-1} \frac{(x-a)^{r}}{r !} b_{r}+\lambda_{1} L^{-1}\left[\int_{a}^{x} \frac{1}{\xi_{k}(x)} K_{1}(x, t) G_{1}\left(Z_{n}(t)\right) d t\right] \\
& +\lambda_{2} L^{-1}\left[\int_{a}^{b} \frac{1}{\xi_{k}(x)} K_{2}(x, t) G_{2}\left(Z_{n}(t)\right) d t\right]-L^{-1}\left[\sum_{j=0}^{k-1} \frac{\xi_{j}(x)}{\xi_{k}(x)} Z^{(j)}(x)\right]
\end{aligned}
$$

Such that,

$$
\begin{aligned}
& L^{-1}\left[\int_{a}^{x} \frac{1}{\xi_{k}(x)} K_{1}(x, t) G_{1}\left(Z_{n}(t)\right) d t\right]=\int_{a}^{x} \frac{(x-t)^{k}}{k ! \xi_{k}(x)} K_{1}(x, t) G_{1}\left(Z_{n}(t)\right) d t \\
& \sum_{j=0}^{k-1} L^{-1}\left[\frac{\xi_{j}(x)}{\xi_{k}(x)}\right] Z^{(j)}(x)=\sum_{j=0}^{k-1} \int_{a}^{x} \frac{(x-t)^{k-1} \xi_{j}(t)}{k-1 ! \xi_{k}(t)} Z^{(j)}(t) d t
\end{aligned}
$$

We set,

$$
\Psi(x)=L^{-1}\left[\frac{f(x)}{\xi_{k}(x)}\right]+\sum_{r=0}^{k-1} \frac{(x-a)^{r}}{r !} b_{r} .
$$

Before starting and proving the main results, we introduce the following hypotheses:

(H1) There exist two constants $\alpha, \beta$ and $\gamma_{j}>0, j=0,1, \cdots, k$ such that, for any $Z_{1}, Z_{2} \in C(J, \mathbb{R})$

$$
\begin{gathered}
\left.\left.\mid G_{1}\left(Z_{1}\right)\right)-G_{1}\left(Z_{2}\right)\right)|\leq \alpha| Z_{1}-Z_{2} \mid \\
\left|G_{2}\left(Z_{1}\right)-G_{2}\left(Z_{2}\right)\right| \leq \beta\left|Z_{1}-Z_{2}\right| \\
\left|D^{j}\left(Z_{1}\right)-D^{j}\left(Z_{2}\right)\right| \leq \gamma_{j}\left|Z_{1}-Z_{2}\right|,
\end{gathered}
$$

we suppose that the nonlinear terms $\left.\left.G_{1}(Z(x))\right), G_{2}(Z(x))\right)$ and $D^{j}(Z)=$ $\left(\frac{d^{j}}{d x^{j}}\right) Z(x)=\sum_{i=0}^{\infty} \gamma_{i_{j}}$, ( $D^{j}$ is a derivative operator $), j=0,1, \cdots, k$, are Lipschitz continuous.

(H2) Suppose that for all $a \leq t \leq x \leq b$, and $j=0,1, \cdots, k$ :

$$
\begin{array}{rlrl}
\left|\frac{\lambda_{1}(x-t)^{k} K_{1}(x, t)}{k ! \xi_{k}(x)}\right| & \leq \theta_{1}, & \left|\frac{\lambda_{1}(x-t)^{k} K_{1}(x, t)}{k !}\right| \leq \theta_{2}, \\
\left|\frac{(x-t)^{k-1} \xi_{j}(t)}{(k-1) ! \xi_{k}(t)}\right| & \leq \theta_{3}, \quad\left|\frac{(x-t)^{k-1} \xi_{j}(t)}{(k-1) !}\right| \leq \theta_{4}, \\
\left|\lambda_{2} L^{-1}\left[\frac{K_{2}(x, t)}{\xi_{k}(x)}\right]\right| & \leq \theta_{5}, \quad\left|\lambda_{2} L^{-1}\left[K_{2}(x, t)\right]\right| \leq \theta_{6},
\end{array}
$$


(H3) There exist three functions $\theta_{3}^{*}, \theta_{4}^{*}$, and $\gamma^{*} \in C\left(D, \mathbb{R}^{+}\right)$, the set of all positive function continuous on $D=\{(x, t) \in \mathbb{R} \times \mathbb{R}: a \leq t \leq x \leq b\}$ such that:

$$
\theta_{3}^{*}=\max \left|\theta_{3}\right|, \quad \theta_{4}^{*}=\max \left|\theta_{4}\right|, \quad \text { and } \gamma^{*}=\max \left|\gamma_{j}\right| .
$$

(H4) $\Psi(x)$ is bounded function for all $x$ in $J=[a, b]$.

Theorem 3.1 Assume that (H1)-(H4) hold. If

$$
0<\psi=\left(\alpha \theta_{1}+\beta \theta_{5}+k \gamma^{*} \theta_{3}^{*}\right)(b-a)<1 .
$$

Then there exists a unique solution $Z(x) \in C(J)$ to IVP (1) - (2).

Proof. Let $Z_{1}$ and $Z_{2}$ be two different solutions of IVP (1) - (2)., then

$$
\begin{aligned}
\left|Z_{1}-Z_{2}\right|= & \left.\mid \int_{a}^{x} \frac{\lambda_{1}(x-t)^{k} K_{1}(x, t)}{\xi_{k}(x) k !}\left[G_{1}\left(Z_{1}\right)-G_{1}\left(Z_{2}\right)\right)\right] d t \\
& \left.+\int_{a}^{b} \lambda_{1} L^{-1}\left[\frac{K_{2}(x, t)}{\xi_{k}(x)}\right]\left[G_{2}\left(Z_{1}\right)-G_{2}\left(Z_{2}\right)\right)\right] d t \\
& \left.-\sum_{j=0}^{k-1} \int_{a}^{x} \frac{(x-t)^{k-1} \xi_{j}(t)}{\xi_{k}(t)(k-1) !}\left[D^{j}\left(Z_{1}\right)-D^{j}\left(Z_{2}\right)\right)\right] d t \mid \\
\leq & \left.\int_{a}^{x}\left|\frac{\lambda_{1}(x-t)^{k} K_{1}(x, t)}{\xi_{k}(x) k !}\right| \mid G_{1}\left(Z_{1}\right)-G_{1}\left(Z_{2}\right)\right) \mid d t \\
& +\int_{a}^{b}\left|\lambda_{1} L^{-1}\left[\frac{K_{2}(x, t)}{\xi_{k}(x)}|| G_{2}\left(Z_{1}\right)-G_{2}\left(Z_{2}\right)\right)\right| d t \\
& \left.-\sum_{j=0}^{k-1} \int_{a}^{x}\left|\frac{(x-t)^{k-1} \xi_{j}(t)}{\xi_{k}(t)(k-1) !}\right| \mid D^{j}\left(Z_{1}\right)-D^{j}\left(Z_{2}\right)\right) \mid d t \\
\leq & \left(\alpha \theta_{1}+\beta \theta_{5}+k \gamma^{*} \theta_{3}^{*}\right)(b-a)\left|Z_{1}-Z_{2}\right|,
\end{aligned}
$$

we get $(1-\psi)\left|Z_{1}-Z_{2}\right| \leq 0$. Since $0<\psi<1$, so $\left|Z_{1}-Z_{2}\right|=0$, therefore, $Z_{1}=Z_{2}$ and the proof is completed.

Theorem 3.2 Suppose that (H1)-(H4), and if $0<\psi<1$, hold, the series solution $Z(x)=\sum_{m=0}^{\infty} Z_{m}(x)$ and $\left\|Z_{1}\right\|_{\infty}<\infty$ obtained by the m-order deformation is convergent, then it converges to the exact solution of the Volterra-Fredholm integro-differential equation (1) - (2).

Proof. Denote as $(C[0,1],\|\cdot\|)$ the Banach space of all continuous functions on $J$, with $\left|Z_{1}(x)\right| \leq \infty$ for all $x$ in $J$.

Frist, we define the sequence of partial sums $s_{n}$, let $s_{n}$ and $s_{m}$ be arbitrary partial sums with $n \geq m$. We are going to prove that $s_{n}=\sum_{i=0}^{n} Z_{i}(x)$ is a Cauchy sequence in 
this Banach space:

$$
\begin{aligned}
\left\|s_{n}-s_{m}\right\|_{\infty}= & \max _{\forall x \in J}\left|s_{n}-s_{m}\right|=\max _{\forall x \in J}\left|\sum_{i=0}^{n} Z_{i}(x)-\sum_{i=0}^{m} Z_{i}(x)\right|=\max _{\forall x \in J}\left|\sum_{i=m+1}^{n} Z_{i}(x)\right| \\
= & \max _{\forall x \in J} \mid \sum_{i=m+1}^{n} \int_{a}^{x} \frac{\lambda_{1}(x-t)^{k} K_{1}(x, t)}{k ! \xi_{k}(x)} A_{i-1} d t+\sum_{i=m+1}^{n} \int_{a}^{b} \lambda_{2} L^{-1}\left[\frac{K_{2}(x, t)}{\xi_{k}(x)}\right] B_{i-1} d t \\
& -\sum_{j=0}^{k-1} \int_{a}^{x} \frac{\xi_{j}(t)(x-t)^{k-1}}{(k-1) ! \xi_{k}(t)} \gamma_{(i-1)} d t \mid \\
= & \max _{\forall x \in J} \mid \int_{a}^{x} \frac{\lambda_{1}(x-t)^{k} K_{1}(x, t)}{k ! \xi_{k}(x)}\left(\sum_{i=m}^{n-1} A_{i}\right) d t+\int_{a}^{b} \lambda_{2} L^{-1}\left[\frac{K_{2}(x, t)}{\xi_{k}(x)}\right]\left(\sum_{i=m}^{n-1} B_{i}\right) d t \\
& -\sum_{j=0}^{k-1} \int_{a}^{x} \frac{\xi_{j}(t)(x-t)^{k-1}}{(k-1) ! \xi_{k}(t)}\left(\sum_{i=m}^{n-1} \gamma_{i_{J}} d t\right) \mid .
\end{aligned}
$$

From (6), we have

$$
\sum_{i=m}^{n-1} A_{i}=G_{1}\left(s_{n-1}\right)-G_{1}\left(s_{m-1}\right), \sum_{i=m}^{n-1} B_{i}=G_{2}\left(s_{n-1}\right)-G_{2}\left(s_{m-1}\right), \sum_{i=m}^{n-1} \gamma_{i}=D^{j}\left(s_{n-1}\right)-D^{j}\left(s_{m-1}\right) .
$$

So,

$$
\begin{aligned}
\left\|s_{n}-s_{m}\right\|_{\infty}= & \max _{\forall x \in J} \mid \int_{0}^{x} \frac{\lambda_{1}(x-t)^{k} K_{1}(x, t)}{k ! \xi_{k}(x)}\left[G_{1}\left(s_{n-1}\right)-G_{1}\left(s_{m-1}\right)\right] d t \\
& +\int_{a}^{b} \lambda_{2} L^{-1}\left[\frac{K_{2}(x, t)}{\xi_{k}(x)}\right]\left[G_{2}\left(s_{n-1}\right)-G_{2}\left(s_{m-1}\right)\right] d t \\
& -\sum_{j=0}^{k-1} \int_{a}^{x} \frac{\xi_{j}(t)(x-t)^{k-1}}{(k-1) ! \xi_{k}(t)}\left[D^{j}\left(s_{n-1}\right)-D^{j}\left(s_{m-1}\right)\right] d t \mid \\
\leq & \max _{\forall x \in J} \int_{0}^{x}\left|\frac{\lambda_{1}(x-t)^{k} K_{1}(x, t)}{k ! \xi_{k}(x)}\right|\left|G_{1}\left(s_{n-1}\right)-G_{1}\left(s_{m-1}\right)\right| d t \\
& +\int_{a}^{b}\left|\lambda_{2} L^{-1}\left[\frac{K_{2}(x, t)}{\xi_{k}(x)}\right]\right|\left|G_{2}\left(s_{n-1}\right)-G_{2}\left(s_{m-1}\right)\right| d t \\
& +\sum_{j=0}^{k-1} \int_{a}^{x}\left|\frac{\xi_{j}(t)(x-t)^{k-1}}{(k-1) ! \xi_{k}(t)} \| D^{j}\left(s_{n-1}\right)-D^{j}\left(s_{m-1}\right)\right| d t
\end{aligned}
$$

Let $n=m+1$, then

$$
\left\|s_{n}-s_{m}\right\|_{\infty} \leq \psi\left\|s_{m}-s_{m-1}\right\|_{\infty} \leq \psi^{2}\left\|s_{m-1}-s_{m-2}\right\|_{\infty} \leq \cdots \leq \psi^{m}\left\|s_{1}-s_{0}\right\|_{\infty},
$$

so,

$$
\begin{aligned}
\left\|s_{n}-s_{m}\right\|_{\infty} & \leq\left\|s_{m+1}-s_{m}\right\|_{\infty}+\left\|s_{m+2}-s_{m+1}\right\|_{\infty}+\cdots+\left\|s_{n}-s_{n-1}\right\|_{\infty} \\
& \leq\left[\psi^{m}+\psi^{m+1}+\cdots+\psi^{n-1}\right]\left\|s_{1}-s_{0}\right\|_{\infty} \\
& \leq \psi^{m}\left[1+\psi+\psi^{2}+\cdots+\psi^{n-m-1}\right]\left\|s_{1}-s_{0}\right\|_{\infty} \\
& \leq \psi^{m}\left(\frac{1-\psi^{n-m}}{1-\psi}\right)\left\|Z_{1}\right\|_{\infty} .
\end{aligned}
$$


Since $0<\psi<1$, we have $\left(1-\psi^{n-m}\right)<1$, then $\left\|s_{n}-s_{m}\right\|_{\infty} \leq \frac{\psi^{m}}{1-\psi}\left\|Z_{1}\right\|_{\infty}$. But $\left|Z_{1}(x)\right|<\infty$, so, as $m \longrightarrow \infty$, then $\left\|s_{n}-s_{m}\right\|_{\infty} \longrightarrow 0$.

We conclude that $s_{n}$ is a Cauchy sequence in $C[0,1]$, therefore $Z=\lim _{n \rightarrow \infty} Z_{n}$.

Theorem 3.3 If problem (1) - (2 has a unique solution, then the solution $Z_{n}(x)$ obtained from the recursive relation (13) using VIM converges when $0<\phi=\left(\alpha \theta_{5}+\right.$ $\left.\beta \theta_{6}+k \gamma^{*} \theta_{4}^{*}\right)(b-a)<1$.

Proof. We have from Eq. (13):

$$
\begin{aligned}
Z_{n+1}(x)-Z(x)= & Z_{n}(x)-Z(x)-\left(L^{-1}\left[\sum_{j=0}^{k} \xi_{j}(x)\left[Z_{n}^{(j)}(x)-Z^{(j)}(x)\right]\right]\right. \\
& -L^{-1}\left[\lambda_{1} \int_{a}^{x} K_{1}(x, t)\left[G_{1}\left(Z_{n}(t)\right)-G_{1}(Z(t))\right] d t\right. \\
& \left.-L^{-1}\left[\lambda_{2} \int_{a}^{b} K_{2}(x, t)\left[G_{2}\left(Z_{n}(t)\right)-G_{2}(Z(t)) d t\right]\right]\right) .
\end{aligned}
$$

If we set, $\xi_{k}(x)=1$, and $W_{n+1}(x)=Z_{n+1}(x)-Z(x), W_{n}(x)=Z_{n}(x)-Z(x)$, since $W_{n}(a)=0$, then

$$
\begin{aligned}
W_{n+1}(x)= & W_{n}(x)+\int_{a}^{x} \frac{\lambda_{1} K_{1}(x, t)(x-t)^{k}}{k !}\left[G_{1}\left(Z_{n}(t)\right)-G_{1}(Z(t))\right] d t \\
& +\int_{a}^{b} \lambda_{2} L^{-1}\left[K_{2}(x, t)\left[G_{2}\left(Z_{n}(t)\right)-G_{2}(Z(t))\right]\right] d t \\
& -\sum_{j=0}^{k-1} \int_{a}^{x} \frac{\lambda_{1} \xi_{j}(t)(x-t)^{k-1}}{(k-1) !}\left[D^{j}\left(Z_{n}(t)\right)-D^{j}(Z(t))\right] d t-\left(W_{n}(x)-W_{n}(a)\right) .
\end{aligned}
$$

Therefore,

$$
\begin{aligned}
\left|W_{n+1}(x)\right| \leq & \int_{a}^{x}\left|\frac{\lambda_{1} K_{1}(x, t)(x-t)^{k}}{k !}\right|\left|W_{n}\right| \alpha d t+\int_{a}^{b}\left|\lambda_{2} L^{-1}\left[K_{2}(x, t)\left|W_{n}\right| \beta\right]\right| d t \\
& +\sum_{j=0}^{k-1} \int_{a}^{x}\left|\frac{\lambda_{1} \xi_{j}(t)(x-t)^{k-1}}{(k-1) !}\right| \max \left|\gamma_{j}\right|\left|W_{n}\right| d t \\
\leq & \left|W_{n}\right|\left[\int_{a}^{x} \alpha \theta_{5} d t+\int_{a}^{b} \beta \theta_{6} d t+\sum_{j=0}^{k-1} \int_{a}^{x} \theta_{4}^{*}|\max | \gamma_{j} \mid\right] \\
\leq & \left|W_{n}\right|\left(\alpha \theta_{5}+\beta \theta_{6}+k \gamma^{*} \theta_{4}^{*}\right)(b-a)=\left|W_{n}\right| \phi
\end{aligned}
$$

Hence,

$$
\left\|W_{n+1}\right\|=\max _{\forall x \in J}\left|W_{n+1}(x)\right| \leq \phi \max _{\forall x \in J}\left|W_{n}(x)\right|=\phi\left\|W_{n}\right\| .
$$

Since $0<\phi<1$, then $\left\|W_{n}\right\| \longrightarrow 0$. So, the series converges and the proof is complete. 


\section{Numerical Results}

Example 1. Consider the following Volterra-Fredholm integro-differential equation.

$$
Z^{\prime \prime}(x)+Z^{\prime}(x)-Z(x)=e^{x-1}-e^{x}-1+\int_{0}^{x} Z(s) d s+\int_{0}^{1} e^{s+x}(Z(s))^{2} d s,
$$

with the initial condition $Z(0)=1, Z^{\prime}(0)=-1$, and the the exact solution is $Z(x)=e^{-x}$.

Table 1: Numerical Results of the Example 1.

\begin{tabular}{lccccc}
\hline $\mathrm{x}$ & Exact solution & ADM & Error ADM & VIM & Error VIM \\
\hline 0.1 & 0.904837418 & 0.896160501 & 0.008676917 & 0.904350694 & 0.000486724 \\
0.2 & 0.818730753 & 0.783594511 & 0.035136242 & 0.817029618 & 0.001701135 \\
0.3 & 0.740818221 & 0.660685557 & 0.080132664 & 0.737405770 & 0.003412451 \\
0.4 & 0.670320046 & 0.525762821 & 0.144557225 & 0.664765171 & 0.005554875 \\
0.5 & 0.606530659 & 0.377106516 & 0.229424144 & 0.598327465 & 0.008203194 \\
0.6 & 0.548811636 & 0.212950800 & 0.335860836 & 0.537264396 & 0.011547240 \\
0.7 & 0.496585303 & 0.031483915 & 0.465101388 & 0.480719900 & 0.015865403 \\
0.8 & 0.449328964 & -0.169154690 & 0.618483654 & 0.427831430 & 0.021497534 \\
0.9 & 0.406569659 & -0.390880529 & 0.797450188 & 0.377751090 & 0.028818569 \\
1.0 & 0.367879441 & -0.635673673 & 1.003553114 & 0.329664630 & 0.038214811 \\
\hline
\end{tabular}

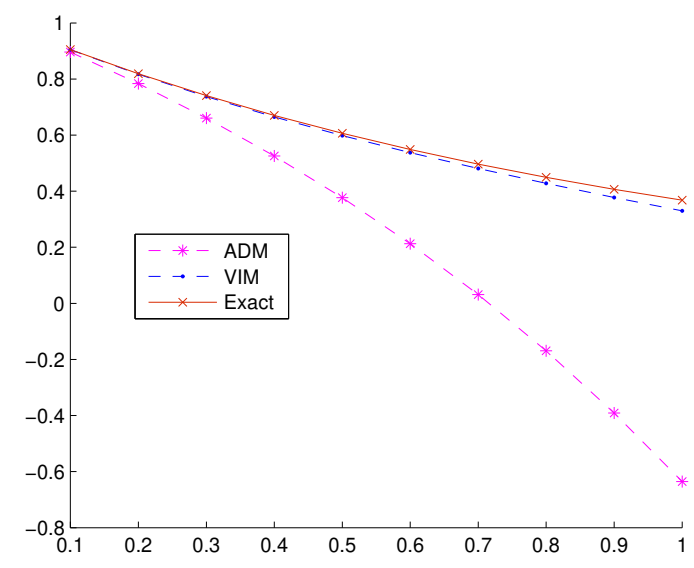

(a) Comparative between ADM, VIM, and Exact solutions

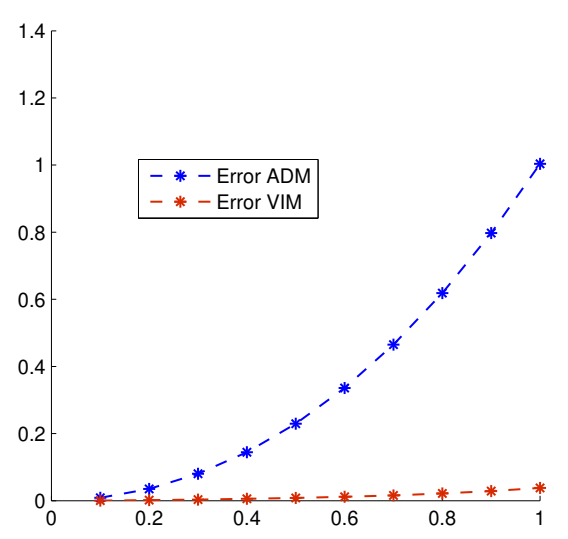

(b) Comparative between Error of ADM , VIM

Figure 1: Numerical Results of the Example 1. 


\section{Comparison Among the Methods}

The comparison among of the methods, it can be seen from the results of the above example:

- The methods are powerful, efficient and give approximations of higher accuracy. Also, they can produce closed-form solutions if they exist.

- Although the results obtained by these methods when applied to Volterra-Fredholm integro-differential equations are the same approximately. VIM is seen to be much easier and more convenient than the ADM.

- One advantage of VIM is that the initial solution can be freely chosen with some unknown parameters. An interesting point about this method is that with few number of iterations, or even in some cases with only one iteration, it can produce a very accurate approximate solution.

- The VIM has a more rapid convergence than the ADM. Also, the number of computations in VIM is less than the ones in ADM.

\section{Conclusions}

We present a comparative study between the Adomian decomposition and variational iteration methods for solving nonlinear Volterra-Fredholm integro-differential equations. From the computational viewpoint, the variational iteration method is more efficient, convenient and easy to use. Moreover, we proved the existence and uniqueness results and convergence of the solution. The methods are very powerful and efficient in finding analytical as well as numerical solutions for wide classes of linear and nonlinear VolterraFredholm integro-differential equations. The convergence theorems and the numerical results establish the precision and efficiency of the proposed techniques.

\section{References}

[1] Abbaoui, K. and Cherruault, Y. Convergence of Adomian's method applied to nonlinear equations, Math. Comput. Modelling, 20(9) (1994), 69-73.

[2] Abbasbandy, S. and Elyas, S. Application of variational iteration method for system of nonlinear Volterra integro-differential equations, Math. Comput. Appl. 2(14) (2009), 147158.

[3] Adomian, G. A review of the decomposition method in applied mathematics, J. Math. Anal. Appl. 135(2) (1988), 501-544.

[4] Alao, S. Akinboro1, F. Akinpelu, F. and Oderinu, R. Numerical solution of integrodifferential equation using Adomian decomposition and variational iteration methods, IOSR J. Math. 10(4) (2014), 18-22.

[5] Bani Issa, M., Hamoud, A. and Ghadle, K. Numerical solutions of fuzzy integro-differential equations of the second kind, J. Math. Computer Sci. 23 (2021), 67-74.

[6] Behzadi, S., Abbasbandy, S., Allahviranloo, T. and Yildirim, A. , Application of homotopy analysis method for solving a class of nonlinear Volterra-Fredholm integro-differential equations, J. Appl. Anal. Comput. 2(2) (2012), 127-136.

[7] Boichuk, A.A., Kozlova, N.A. and Feruk, V.A., Weakly nonlinear integral equations of the Hammerstein type, Nonlinear Dyn. Syst. Theory, 19(2) (2019), 289-301. 
[8] Dawood, L., Hamoud, A. and Mohammed, N. Laplace discrete decomposition method for solving nonlinear Volterra-Fredholm integro-differential equations, J. Math. Computer Sci. 21(2) (2020), 158-163.

[9] Hamoud, A.A. and Ghadle, K.P. Some new existence, uniqueness and convergence results for fractional Volterra-Fredholm integro-differential equations, J. Appl. Comput. Mech. 5(1) (2019), 58-69.

[10] Hamoud, A. and Ghadle, K. Usage of the homotopy analysis method for solving fractional Volterra-Fredholm integro-differential equation of the second kind, Tamkang J. Math., 49(4) (2018), 301-315.

[11] Hamoud, A. and Ghadle, K. The approximate solutions of fractional Volterra-Fredholm integro-differential equations by using analytical techniques, Probl. Anal. Issues Anal., 7(25) (2018), 41-58.

[12] Hamoud, A.A. and Ghadle, K.P. Modified Adomian decomposition method for solving fuzzy Volterra-Fredholm integral equations, J. Indian Math. Soc. 85(1-2) (2018), 52-69.

[13] He, J.H. A variational approach to nonlinear problems and its application, Mech. Applic. 20(1) (1998), 30-34.

[14] Hussain, K., Hamoud, A. and Mohammed, N. Some new uniqueness results for fractional integro-differential equations, Nonlinear Funct. Anal. Appl., 24(4) (2019), 827-836.

[15] Mittal, R. and Nigam, R. Solution of fractional integro-differential equations by Adomian decomposition method, Int. J. Appl. Math. Mech. 4(2) (2008), 87-94.

[16] Wazwaz, A.M. Linear and Nonlinear Integral Equations Methods and Applications, Springer Heidelberg Dordrecht London New York, 2011.

[17] Yang, C. and Hou, J. Numerical solution of integro-differential equations of fractional order by Laplace decomposition method, Wseas Trans. Math. 12(12) (2013), 1173-1183. 